\title{
NONLINEAR ELASTIC WAVES IN A GRANULAR MEDIUM
}

\author{
A.N. BOGDANOV and A.T. SKVORTSOV \\ N.N. Andreev Acoustical Inst., Academy of Sciencies of USSR, Shvernika st. 4, Moscow 117036, \\ USSR
}

The propagation and interaction of elastic waves in the granular medium with regard for its nonlinear properties is considered. This medium is represented by the cubic packing of elastic spheres. The obtained equations allow to describe the nonlinear compression and shear waves in the simple model of granular medium.

La propagation et 1 'interaction des ondes élastigue daris le milieu granuleux avec le compte des propriétés nonlineaires est regardé. Ce milieu est représenté par le paquet cubigue des sphéres elastigues. Les équations obtenues permettent decrire des ondes nonlineaires de la compression et du déplacement dans le modéle simple du milieu granuleux.

The problem of propagation of the sound waves in a granular media is widely investigated in seismoacoustics $/ 1,2 /$ (the model of the structurally nonuniform media) and in composite mechanics $/ 3,4,5 /$ (nonlinear vibroabsorbing covers). In present work the main nonlinear phenomena are considered, occurring in propagation of the elastic waves in the simple model of a granular medium: uniformly compressed cubic packing of the contacting spheres.

\section{Propagation of the elastic waves in two-conponent granular nediun}

We assume, that the granular medium in this case is represented by the cubic packing of subsequently alternating layers of two types /6/. Each layer consists of same spheres and spheres in different layers differ from each another in seize and material properties (density, Young's modulus, Poisson's ratio)(Fig.1). 


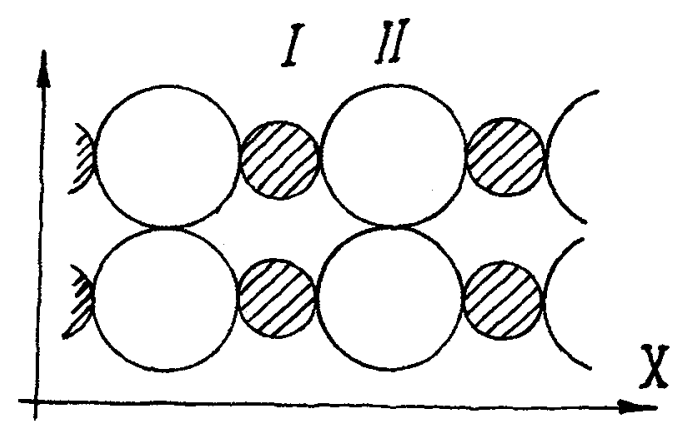

Fig. 1 - I and II are two types of spheres.

In addition, there is supposed, that the medium is subjected to the pressure $\mathrm{p}$ and the longitudinal waves propagate perpendicularly to the layers. Thus the problem comes to one-dimensional. The system of wave equations may be obtained analogously to that in /4/ in longwave approximation.

This system in the linear case is easily shown /6/ to be characterized by presence of two types of vibration /7/: low-frequency acoustic (with the dispersion law $\omega^{2}=c_{0}^{2} k^{2}-\beta^{2} k^{4}, c_{0}$ - sound velocity) and high-frequency optical $\left(\omega^{2}=\omega_{0}^{2}-c_{0}^{2} k^{2}\right)$. Taking into account the nonlinear terms, the obtained system describes a different nonlinear interactions of low- and high-frequency waves (nonlinear attenuation, parametric interaction, propagation of solitons etc. $/ 6 / 6$. In particular, the separating of low- and high-frequency components of perturbation by means of the special procedure /8/ gives equations, analogous to Zakharov equations in plasma /9/:

$$
\begin{aligned}
& u_{t t}^{2}-c_{0}^{2} u_{x x}-\beta^{2} u_{x x x x}=-2 u_{x} u_{x x}-c_{1} \cdot\left(|A|^{2}\right)_{x} \\
& i w_{0} A_{t}+c_{0}^{2} A_{x x}=c_{2} A_{x}, c_{1}, c_{2}, x>0
\end{aligned}
$$

In this case, Eq.(1) describes low-frequency perturbation and Eg. (2) describes the envelope of high-frequency oscillation. For example, in the case of purely acoustic waves ( $A=0$ ) Eq. (1) takes the form of Boussinesq's equation, solutions of which have been studied well / $/ 8$. In opposite case of high-frequency oscillation Eg.(2) comes to nonlinear scrödinger equation $/ 6 /$ : 


$$
i \omega_{O} A_{t}+c_{O}^{2} A_{X X}-\frac{c_{1} c_{2}}{c_{O}^{2}}|A|^{2} A=0
$$

The signs of the coefficients of second and third terms in Eg. (3) show, that perturbations don't propagate in the form of soliton (Lighthill criterion /10/), and hence, the periodic oscillations, described by Eq.(3), is stable with respect to the self-modulation.

\section{Nonlinear shear waves in a granular nedium}

Following $/ 1 /$, there is considered the propagation of shear waves in the granular medium, represented by the cubic packing of elastic spheres of the diameter $a$ under pressure $p$. For the case of quasi-static deformations the analysis of tension in the medium may be implemented on the basis of the results of the applied mechanics $11,12 /$, in which the nonlinear character of tangential force of the interaction of contacting spheres with respect to the transverse deflection has been determined. This dependence in quadratic approximation has the form /12/:

$$
\mathbf{F}=\gamma_{1} \varepsilon-\gamma_{2} \varepsilon|\varepsilon|
$$

where $\gamma_{1}$ and $\gamma_{2}$ are defined by sphere seize and material and pressure. In this limitation, there is obtained the system of equations of transverse motion of the granular medium, which may be transformed in the longwave approximation to the system of two nonlinear differential equations /13/. By standard method it may be shown, that in linear case the system of wave equations so that in pt. 1 is characterized by two types of oscillation: low- and high-frequency (the dispersion laws $\omega_{1}^{2}=c_{t}^{2} k^{2}\left(1+(k a)^{2} / 60\right), \omega_{2}^{2}=\omega_{0}^{2}-4 c_{t}^{2} k^{2}$, respectively, where $c_{t}$ is the velocity of shear waves in the granular medium).

The whole system (with its nonlinear terms) allows to consider a different processes of waves interaction /13/. For example, it is easy to show, that for low-frequency soliton-like (unipolar) perturbations the system transfers to well-known Boussinesq's equation $/ 8 /$ :

$$
v_{t t}-c_{t}^{2} v_{x x}+c_{t}^{2} \frac{a^{2}}{60} v_{x x x x}=\left\{\begin{array}{l}
-\delta v_{x} v_{x x}, v_{x}>0 \\
\delta v_{x} v_{x x}, v_{x}<0
\end{array}\right.
$$

where $\delta>0, V$ describes the local transverse displacement of the medium 
particles. The stationary solutions of $\mathrm{Eg} .(5)$ have the form of solitary waves:

$$
\nabla_{x}=\nabla_{O x} c h^{-2}\left(\left(x-v_{1} t\right) / \Delta\right) \text {, }
$$

where $\nabla_{O x}, \Delta, V_{1}$ are the amplitude, the width and the velocity of the soliton, respectively. Due to the form of the nonlinear term in Eq. (5) the soliton velocity $V_{1}$ is always less than shear wave velocity $c_{t}$. The obtained results for low-frequency waves (when Gassman theory is adequate) may be generalized for the case of granular medium, in which the inter-sphere space is filled by fluid or by water-like material (rubber) for the case of composite. Then the influence of filling comes to the respective simple renorming of density and elasticity /1/.

\section{References}

/1/ J.E.WHITE. Underground sound. Application of seismic waves. Amsterdam-Oxford-NY 1983.

12/ K.A.NAUGOL'NYKH, L.A.OSTROVSKY. Non-linear wave processes in acoustics. Moscow: Nauka. 1990.

/3/ J.M.BRETTELL. Acustica. 65 (1987) $149-51$.

/4/ V.F.nESTERENRo. Zh. Prikl. Mekh. Tekh. Phys. USSR 5 (1983) 136-148. (in Russian, English trans. in J. Appl. Mech. Tech. Phys.)

/5/ A.N.LAZARIDI, V.F.NESTERENKO. where the previous. 3 (1985) 115-118.

16) A.N.BOGDaNOV, A.T.SKVORTSOV. Proc. of XI All-Union Acoust. conf. Moscow $1981 \mathrm{sec}$. B 39-41. (in Russian).

$/ 7 /$ N.W.ASHCROFT, N.D.MERMIN. Solid state physics. Cornell University 1971 V.2.

/8/ R.K.DODD, J.C.EILBECK, J.D.GIBBON, H.C.MORRIS. Solitons and nonlinear wave equations. Academic Press 1982.

19/ V.E.ZAKHAROV. Zh. Exp. Tekh. Phys. USSR 62 (1972) 5 1745-59. (in Russian).

$/ 10 /$ G.B.WHITHAM. Linear and nonlinear waves. NY 1974.

$/ 11 / J$.DUFFY, R.D.MINDLIN. J. Appl. Mech. 24 (1957) 4 585-593.

/12/R.D.MINDLIN. J. App 1. Mech.,Trans. ASME. 71 (1949) A259-68.

/13/A.N.BOGDANOV, A.T.SRVORTSOV. SOV. PhYs. Acoust. (to be published). 